\title{
RESILIENCIA Y RENDIMIENTO ACADÉMICO EN ESTUDIANTES DEL 5TO Y 6TO GRADO DE PRIMARIA
}

\author{
RESILIENCE AND ACADEMIC PERFORMANCE IN FIFTH AND SIX PRIMARY \\ SCHOOL STUDENT
}

\author{
Rossina Gallesi G. \\ María Matalinares $C$. \\ Universidad Nacional Mayor de San Marcos, Lima, Perú \\ (RECIBIDO EL 15/2/2012; ACEPTADO 15/6/2012)
}

\begin{abstract}
RESUMEN
El objetivo del estudio fue determinar la relación entre los factores personales de resiliencia y el rendimiento académico. La muestra estuvo conformada por 202 estudiantes de 5 . to y 6 . to grado de educación primaria de la Ciudad Satélite del Callao, de ambos sexos, cuyas edades fluctúan entre los 9 y 14 años; a quienes se aplicó el Inventario de Factores Personales de Resiliencia propuesto por Salgado (2005) y se evaluó el rendimiento académico a través de las calificaciones en las asignaturas más significativas y las notas promedio del semestre. Los resultados mostraron que existe relación significativa entre los factores personales de resiliencia y el rendimiento académico. Se encuentra que los factores personales Autoestima y Empatía presentan correlación significativa con todas las áreas de rendimiento académico. Autonomía con Comunicación, Lógico Matemático y Personal Social. Humor con Comunicación, Ciencia-Ambiente y Personal Social y Creatividad con Personal Social. Resiliencia presenta correlación significativa con todas las áreas de rendimiento académico. En Empatía, Creatividad y Resiliencia se encontraron diferencias significativas a favor de las mujeres. En Autoestima, Autonomía Creatividad y Resiliencia se encontraron diferencias significativas a favor de los alumnos de 5. to Grado. Las mujeres tienen un rendimiento académico significativamente más alto que los varones en Comunicación, Ciencia y Ambiente y Personal Social.
\end{abstract}

Palabras claves: factores personales de resiliencia, rendimiento académico, niños. 


\begin{abstract}
The aim of this study was to determine the relationship between personal factors of resilience and academic achievement. The sample consisted of 202 students from 5th. and 6th. Grade of primary education, both sexes, whose ages range between 9 and 14, who completed the Inventory of Personal Resilience Factors proposed by Salgado (2005) and academic performance was assessed through the qualifications in the subjects more significant and the average grades of the semester. The results showed a significant relationship between personal factors of resilience and academic achievement. It is found that: personal factors esteem and Empathy have significant correlation with all areas of academic achievement. Communication, Mathematical Logic and Personal Social. Humor with communication, Science, Environment and Social Personal with Creativity. Resilience has significant correlation with all areas of academic achievement. In Empathy, Creativity and Resilience significant differences were found for women. In Self-Esteem, Creativity and Resilience Autonomy significant differences were found for students in 5th. Degree. Women have a significantly higher academic achievement than boys in Communication, Science and Environment and social Personal.
\end{abstract}

Keywords: factors of resilience, academic achievement, children.

\title{
INTRODUCCIÓN
}

Rutter (1991), citado Melillo y Suárez (2001), entiende por resiliencia una respuesta global en la que se ponen en juego los mecanismos de protección, entendiendo por estos no la valencia contraria a los factores de riesgo, sino aquella dinámica que permite al individuo salir fortalecido de la adversidad, en cada situación específica, respetando las características personales.

A decir de Rutter (1999), en Melillo y Suárez (2001), existe una gran variedad de respuestas de los niños para describir la resistencia a experiencias de riesgo psicosocial. Existen múltiples factores de protección frente al riesgo; así los niños varían en su vulnerabilidad al estrés psicosocial y la adversidad según factores genéticos o ambientales; las experiencias del ambiente familiar inciden en las diferencias individuales de los niños la reducción de las reacciones negativas y aumento de las positivas, influyen en la medida en que los efectos de la adversidad persisten en el tiempo; las experiencias nuevas puede proporcionar oportunidades para un "punto de inflexión" sobre los efectos; las experiencias positivas aunque en sí mismas no ejercen un gran efecto de protección pueden ser útiles para neutralizar algunos factores de riesgo; y que el procesamiento cognitivo y afectivo de experiencias puede influir en la resistencia que se desarrolla.

De acuerdo con Vanistendael (1997), en Kalawski y Has (2003) existen cinco dimensiones de la resiliencia:

A. Existencia de redes sociales informales: la persona tiene amigos, participa de actividades con ellos y lo hace con agrado; tiene en general una buena relación con los adultos.

B. Sentido de vida, trascendencia: la persona muestra capacidad para descubrir un sentido y una coherencia en la vida. 
C. Autoestima positiva: la persona se valora a sí misma, confía en sus capacidades y muestra iniciativa para emprender acciones o relaciones con otras personas porque se siente valiosa y merecedora de atención.

D. Presencia de aptitudes y destrezas: es capaz de desarrollar sus competencias y confiar en ellas.

E. Sentido del humor: la persona es capaz de jugar, reír, gozar de las emociones positivas y de disfrutar de sus experiencias.

En el Perú, Escudero (2007) desarrolló un estudio a fin de establecer la relación entre la resiliencia y el sentido de vida (religiosidad intrínseca y objetivos vitales) en adolescentes de 4to. y 5to. de secundaria; trabajó con una muestra de 369 adolescentes de ambos sexos entre 14 y 19 años de los colegios nacionales. Encontró relación significativa entre resiliencia y religiosidad, resiliencia y objetivos vitales, siendo los objetivos vitales los que mejor explican las diferencias en cuanto a resiliencia y de entre estos la subdimensión de desarrollo personal la que tiene mayor importancia. Flores (2008) investiga la resiliencia y el proyecto de vida en estudiantes del tercer año de secundaria de colegios nacionales. Los resultados mostraron que existe relación altamente significativa entre el grado de resiliencia y el grado de definición del proyecto de vida, tanto en la muestra de estudiantes varones como en la de mujeres. Bulnes, Ponce, Huerta, Álvarez, Santibáñez, Atalaya, Aliaga y Morocho (2008) realizaron un estudio descriptivo correlacional y comparativo de resiliencia y estilos de socialización parental en 394 estudiantes de 4to. y 5to. año de secundaria de Lima Metropolitana. Los resultados mostraron una correlación significativa entre ambas variables tomando en cuenta género y tipo de colegio. Flores (2008) realizó un estudio sobre resiliencia y proyecto de vida en estudiantes del tercer año de secundaria con una muestra de 400 estudiantes de ambos sexos cuya edades fluctúan entre 13 a18 años del 3. ${ }^{\text {ro }}$ de secundaria de 6 colegios estatales y particulares de la UGEL 03 de Lima. Los resultados mostraron que existe relación entre el grado de resiliencia y el grado de definición del proyecto de vida, tanto en varones como en mujeres. Quintana, Montgomery y Malaver (2009), los modos de afrontamiento y conducta resiliente en adolescentes espectadores de violencia entre pares Los datos indican que existen diferencias significativas en ciertos modos de afrontamiento, en la conducta resiliente, y entre esta y la mayoría de los modos de afrontamiento manifestados por los distintos tipos de espectadores de violencia entre pares. Astete, Benavides y Palacios (2009) realizaron un estudio descriptivo con el objetivo de identificar el nivel de resiliencia en niños de 8 a 12 años provenientes de familias disfuncionales. Los resultados mostraron que los niños presentan un alto nivel de resiliencia según los componentes resilientes: competencia social, resolución de problemas y autonomía y sentido de propósito y de futuro. Aponte (2009), en el estudio realizado "Relación entre estilo de crianza parental y resiliencia adolescente en la conducta violenta", realizado en 312 estudiantes de secundaria de ambos sexos de Lima, mostró la existencia de correlación inversa entre la resiliencia y la conducta violenta con independencia del estilo parental. Velásquez y Montgomery (2009) 
realizan un estudio sobre resiliencia y depresión en estudiantes de secundaria de Lima Metropolitana con participación en actos violentos y sin ellas; los resultados muestran que existen correlaciones positivas en los componentes internos de ambas pruebas, mientras que al correlacionarlas entre ellas la relación es más diversa. Se identificaron diferencias entre los alumnos violentos y no violentos en cuanto a los factores de confianza, autoritarismo e ideas suicidas; así mismo, se identificaron diferencias entre mujeres y varones en el control emocional, toma de decisiones, autocrítica, propensión al llanto y otras variables. Salgado (2011) en el estudio Felicidad, resiliencia y optimismo en estudiantes de colegios nacionales de la ciudad de Lima, con una muestra de 275 estudiantes de 15 a 18 años, de 5to. año de secundaria de dos colegios nacionales mixtos de Lima, encontró que existe una relación altamente significativa, a mayor resiliencia, mayor optimismo y mayor felicidad. Por último, Matalinares, Arenas, Yaringaño, Sotelo, Sotelo, Díaz, Dioses, Ramos, Mendoza, Medina, Pezua, Muratta, Pareja y Tipacti (2011) realizaron una investigación con 420 estudiantes, de ambos sexos, cuyas edades fluctuaban entre 10 y 12 años, que cursaban el quinto y sexto grado de primaria, procedentes de diferentes centros educativos estatales de Lima Metropolitana. Los resultados mostraron que existe correlación entre los factores personales de resiliencia y autoconcepto; además reportaron una relación entre los factores autoestima y empatía con todos los componentes del autoconcepto. Al comparar el autoconcepto de varones con mujeres se encontró diferencias significativas en los componentes físico, social y familiar a favor de las mujeres. Por último, al comparar varones y mujeres en los factores de resiliencia se encontró diferencias significativas a favor de las mujeres en autoestima, empatía y resiliencia.

Ahora bien, respecto de la variable rendimiento académico, Adell (2006) explica que es un constructo complejo y viene determinado por un gran número de variables y las correspondientes interacciones de muy diversos referentes: inteligencia, motivación, personalidad, actitudes, contexto, etc. El mismo autor explica que las variables predictoras del rendimiento académico son en el ámbito personal: el sexo y nivel, la existencia o no de problemas sensoriales, salud y fitness, autoconcepto, actitud ante los valores, esfuerzo-éxito, confianza en el futuro, valoración trabajo intelectual, aspiración nivel de estudios; en el ambiente ámbito familiar: el número de hermanos, nivel de estudios de los padres, la ocupación familiar, el nivel económico, la comunicación familiar, las actitudes familiares, las expectativas de estudios hijos, la valoración trabajo intelectual, la ayuda brindada al estudio de los hijos; en el ámbito escolar: la dinámica de la clase, la integración en el grupo, la relación tutorial, el clima de la clase, la participación en el centro; en el comportamiento del alumno: la disposición y el tiempo libre, las actividades culturales, los usos lingüísticos, la televisión-radio-cine-video, el uso de las drogas y alcohol, las experiencias sexuales, las actividades cognitivas y la dedicación y aprovechamiento de los estudios. El ámbito de los resultados: las notas del curso anterior, las notas del curso actual y las notas por asignaturas; por último plantea el ámbito del bienestar: satisfacción con los resultados y la satisfacción con el tiempo libre. No todas las variables han demostrado la misma capacidad de predicción de los rendimientos medidos en notas y bienestar. 
Rodríguez (1982) citado por Adell (2002), explica que las notas son una realidad que se nos impone sobre cualquiera otra, pues las calificaciones constituyen en sí mismas el criterio social y legal del rendimiento en el alumnado. Se podría decir que las notas cumplen, además de una finalidad informativa a padres y autoridades académicas, la función de pronóstico, puesto que ayudan a saber no solo dónde está el alumno en cada momento, sino cuales son sus posibilidades en el futuro. Para Pérez Serrano (1981) citado por Adell (2002), las calificaciones son el mejor criterio con que se cuenta para definir el rendimiento. Por último, Page (1990) citado por Adell (2002) señala que las notas son las medidas más utilizadas por el profesorado y los centros a la hora de valorar el rendimiento de sus alumnos.

\section{Objetivo general del estudio}

Determinar la relación entre los factores personales de resiliencia: autoestima, empatía, autonomía, humor y creatividad con el rendimiento académico en: Comunicación Integral, Lógico Matemático, Personal Social y Ciencia y Ambiente en una muestra de estudiantes de 5to. y 6to. Grado de primaria.

\section{Objetivos específicos}

1. Diagnosticar el nivel de resiliencia obtenido por la muestra de estudiantes.

2. Diagnosticar el nivel obtenido en los factores personales de resiliencia en la muestra de estudiantes.

3. Diagnosticar el rendimiento académico obtenido por la muestra de estudiantes.

4. Comparar los niveles de resiliencia de los estudiantes según la variable sexo.

5. Comparar los factores personales de resiliencia de los estudiantes según la variable sexo.

6. Comparar resiliencia según el variable grado de instrucción en la muestra de estudiantes.

7. Comparar los factores personales de resiliencia según el variable grado de instrucción en la muestra de estudiantes.

8. Comparar el rendimiento académico en las áreas de: Lógico Matemático, Comunicación Integral, Personal Social y Ciencia - Ambiente; según la variable sexo en la muestra de estudiantes.

9. Comparar el rendimiento académico en las áreas de: Lógico Matemático, Comunicación Integral, Personal Social y Ciencia y Ambiente según el variable grado de instrucción en la muestra de estudiantes.

\section{Hipótesis general}

Existe una relación significativa entre los factores personales de resiliencia: autoestima, empatía, autonomía, humor y creatividad con el rendimiento académico en: Comunicación Integral, Lógico Matemático, Personal Social y Ciencia y Ambiente en la muestra de estudiantes. 


\section{Hipótesis específicas}

1. Los estudiantes de 5to. y 6to. grado de primaria obtienen un nivel de resiliencia promedio

2. Los estudiantes de 5to. y 6to. grado de primaria obtienen un nivel promedio en los factores personales de resiliencia.

3. Los estudiantes de 5to. y 6to. grado de primaria obtienen el logro previsto para su grado de instrucción “A” en: Comunicación Integral, Lógico Matemático, Personal Social y Ciencia y Ambiente.

4. Existen diferencias significativas en los niveles de resiliencia de los estudiantes según la variable sexo.

5. Existen diferencias significativas en los factores personales de resiliencia de los estudiantes según la variable sexo.

6. Existen diferencias significativas en los niveles de resiliencia de los estudiantes según la variable grado de instrucción en la muestra de estudiantes.

7. Existen diferencias significativas en los factores personales de resiliencia de los estudiantes según la variable grado de instrucción en la muestra de estudiantes.

8. Existen diferencias significativas en el rendimiento académico en las áreas de: Lógico Matemático, Comunicación Integral, Personal Social y CienciaAmbiente; según la variable sexo en la muestra de estudiantes.

9. Existen diferencias significativas en el rendimiento académico en las áreas de: Lógico Matemático, Comunicación Integral, Personal Social y Ciencia y Ambiente según la variable grado de instrucción en la muestra de estudiantes.

\section{MÉTODO}

Para la realización de esta investigación se empleó el método descriptivo con un diseño correlacional y comparativo. Se evaluó a 146 estudiantes de $5 .^{\text {to }}$ y $6 .^{\text {to }} \mathrm{de}$ primaria, de ambos sexos, cuyas edades fluctúan entre los 9 y 14 años procedentes de centros educativos de la Ciudad Satélite del Callao. Dado el carácter de los variables a estudiar se utilizó la técnica psicométrica y de observación indirecta.

\section{Instrumentos de recolección de datos}

Para evaluar los factores personales de resiliencia se empleó el Inventario de Factores Personales de Resiliencia de Ana Cecilia Ana Salgado (2005); para rendimiento académico se emplean los informes de rendimientos del año lectivo oficialmente emitidos por los colegios.

\section{El inventario de factores personales de resiliencia}

Diseñada por Ana Cecilia Salgado (2005), en base a la propuesta de resiliencia de la Regional Training la prueba mide los factores personales de la resiliencia. El inventario consta de cinco factores: la autoestima, la empatía, la autonomía, 
el humor y la creatividad. La prueba consta de 48 afirmaciones y el sujeto examinado tiene dos opciones de respuesta: sí y no. La administración es en forma colectiva en un tiempo aproximado de 30 minutos. El instrumento tiene validez de contenido por criterio de jueces. La confiabilidad fue realizada a través de tres tipos de análisis: el primero observando el monto de valores perdidos para las variables; luego se presenta la información descriptiva básica en base a medidas de tendencia central, de dispersión y de distribución; luego un examen de diferencias entre variables demográficas a través del ANOVA de dos vías $2 \times 4$, en que las variables fueron género y edad, que fueron examinadas independientemente y en interacción sobre los puntajes de los factores de resiliencia.

\section{Información sobre el rendimiento académico}

Para acceder a los datos de la variable rendimiento académico se empleó la técnica documental accediendo a los promedios de los estudiantes en los cursos de Lógico Matemático, Comunicación, Ciencia y Ambiente y Personal Social, luego de culminar el último trimestre de estudios. Estos datos se obtuvieron a través de los informes de rendimientos del año lectivo oficialmente emitidos por los colegios.

\section{RESULTADOS}

\section{Análisis descriptivo}

A continuación se realiza el análisis descriptivo de los datos obtenidos en base a los estadísticos de tendencia central (media, moda, mediana, desviación típica y varianza)

Tabla 1: Resultados en factores personales de resiliencia

\begin{tabular}{cccccc} 
& Autoe. & Empat. & Auton. & Humor. & Creat. \\
\hline N & 202 & 202 & 202 & 202 & 202 \\
Media & 7,83 & 7,16 & 6,84 & 5,80 & 4,58 \\
Nivel & Medio & Medio & Medio & Medio & Medio \\
Mediana & 8,00 & 7,00 & 7,00 & 6,00 & 4,50 \\
Moda & 9 & 8 & 7 & 6 & 4 \\
Des. Típ. & 1,780 & 1,833 & 1,781 & 1,549 & 1,475 \\
Varianza & 3,169 & 3,361 & 3,172 & 2,398 & 2,175 \\
\hline
\end{tabular}

La Tabla 1 indica que el promedio de los alumnos en Autoestima es 7,83, en Empatía es 7,16, en Autonomía es 6,84; en Humor 5,80, en Creatividad es 4,58 y en Resiliencia 32,21. Se observa además que los datos tienen una menor desviación con respecto a la media en Creatividad $(1,475)$ y una mayor desviación en Empatía $(1,833)$. En los factores personales de resiliencia los estudiantes obtienen un nivel Medio. Estos resultados corroboran la hipótesis específicas 1 y 2. 
Tabla 2. Resultados descriptivos en áreas de rendimiento académico

\begin{tabular}{ccccc} 
& Comunicación I. & Lógico M. & Ciencia y A. & Personal S. \\
\hline N & 202 & 202 & 202 & 202 \\
Media & 2,43 & 2,43 & 2,39 & 2,55 \\
Nivel & $\mathrm{B}$ & $\mathrm{B}$ & $\mathrm{B}$ & $\mathrm{B}$ \\
Mediana & 2,00 & 2,00 & 2,00 & 3,00 \\
Moda & 3 & 3 & 2 & 3 \\
Desv. Típ. &, 644 &, 689 &, 599 &, 646 \\
Varianza &, 415 &, 475 &, 359 &, 417 \\
\hline
\end{tabular}

La Tabla 2, indica que el promedio de los alumnos en Comunicación Integral es 2,43, en Matemática es 2,43, en Ciencia y Ambiente es 2,39; y en Personal Social 2,55. Se observa además que los datos tiene una menor desviación con respecto a la media en Ciencia y Ambiente $(0,599)$ y una mayor desviación en Matemática $(0,689)$. En todas las áreas de rendimiento académico se observa nivel B (En Proceso).

Tabla 3. Resultados en los niveles de resiliencia

\begin{tabular}{ccc}
\hline Nivel & Resiliencia & \\
& $\mathbf{f}$ & $\%$ \\
Muy Bajo & 2 & 1,0 \\
Bajo & 6 & 3,0 \\
Promedio & 110 & 54,5 \\
Alto & 81 & 40,1 \\
Muy Alto & 3 & 1,5 \\
Total & 202 & 100,0 \\
\hline
\end{tabular}

En la Tabla 3, se observa en resiliencia que los estudiantes obtienen $1 \%$ en Nivel Muy bajo, 3,0\% en Nivel bajo, 54,55 en el nivel Promedio, 40,1\% en nivel Alto y $1,5 \%$ en nivel Muy Alto.

Tabla 4. Resultados en factores personales en resiliencia

\begin{tabular}{lllllllllll}
\hline Nivel & $\mathrm{f}$ & $\%$ & $\mathrm{f}$ & $\%$ & $\mathrm{f}$ & $\%$ & $\mathrm{f}$ & $\%$ & $\mathrm{f}$ & $\%$ \\
Bajo & 23 & 11,4 & 22 & 10,9 & 7 & 3,5 & 11 & 5,4 & 14 & 6,9 \\
Medio & 92 & 45,5 & 80 & 39,6 & 118 & 58,4 & 131 & 64,9 & 134 & 66,3 \\
Alto & 87 & 43,1 & 100 & 49,5 & 77 & 38,1 & 60 & 29,7 & 54 & 26,7 \\
Total & 202 & 100,0 & 202 & 100,0 & 202 & 100,0 & 202 & 100,0 & 202 & 100,0 \\
\hline
\end{tabular}

En la Tabla 4, se observa en los factores personales de resiliencia que los estudiantes obtienen con más frecuencia y porcentaje el nivel Medio, a excepción del factor Empatía donde en mayor porcentaje se encuentran en Nivel Alto. 
Tabla 5. Resultados según niveles en áreas de rendimiento académico

\begin{tabular}{lcccccccc}
\hline Nivel & \multicolumn{2}{c}{ Comunicación I. } & \multicolumn{2}{c}{ Lógico M. } & \multicolumn{2}{c}{ Ciencia y A. } & \multicolumn{2}{c}{ Personal S. } \\
& f & \% & f & \% & f & \% & f & \% \\
\hline AD & 14 & 6,9 & 18 & 8,9 & 11 & 5,4 & 12 & 5,9 \\
A & 91 & 45,0 & 85 & 42,1 & 102 & 50,5 & 71 & 35,1 \\
B & 94 & 46,5 & 94 & 46,5 & 88 & 43,6 & 114 & 56,4 \\
C & 3 & 1,5 & 5 & 2,5 & 1 & 0,5 & 5 & 2,5 \\
\hline Total & 202 & 100,0 & 202 & 100,0 & 202 & 100,0 & 202 & 100,0 \\
\hline
\end{tabular}

En la Tabla 5, se observa en las áreas de rendimiento académico que los estudiantes muestran promedios con similares porcentajes tanto en el Nivel B (En proceso) así como también en el nivel A (Logro previsto).

Tabla 6. Resultados en factores personales de resiliencia según sexo

\begin{tabular}{llllllllllll}
\hline & \multicolumn{2}{l}{ Autoestima } & \multicolumn{2}{l}{ Empatía } & \multicolumn{4}{c}{ Autonomía } & \multicolumn{2}{c}{ Humor } & \multicolumn{2}{c}{ Creatividad } \\
& $\mathrm{H}$ & $\mathrm{M}$ & $\mathrm{H}$ & $\mathrm{M}$ & $\mathrm{H}$ & $\mathrm{M}$ & $\mathrm{H}$ & $\mathrm{M}$ & $\mathrm{H}$ & $\mathrm{M}$ \\
$\mathrm{N}$ & 103 & 99 & 103 & 99 & 103 & 99 & 103 & 99 & 103 & 99 \\
Media & 7,62 & 8,04 & 6,62 & 7,73 & 6,71 & 6,97 & 5,66 & 5,95 & 4,30 & 4,87 \\
Nivel & Medio & Alto & Medio & Medio & Medio & Medio & Medio & Medio & Medio & Medio \\
Mediana & 8,00 & 8,00 & 7,00 & 8,00 & 7,00 & 7,00 & 6,00 & 6,00 & 4,00 & 5,00 \\
Desv.Típ & 1,766 & 1,778 & 1,874 & 1,615 & 1,813 & 1746 & 1588 & 1,501 & 1,342 & 1,556 \\
Varianza & 3,120 & 3,162 & 3,512 & 2,609 & 3,287 & 3,050 & 2,521 & 2,253 & 1,801 & 2,421 \\
\hline H=Hombre
\end{tabular}

$\mathrm{H}=$ Hombre, $\mathrm{M}=$ Mujer

En la Tabla 6, se observa que el promedio de las mujeres es mayor que el de los hombres en todos el factores personales de resiliencia.

Tabla 7. Resultados en resiliencia según sexo

\begin{tabular}{lcc}
\hline & & RESILIENCIA \\
& $\mathrm{H}$ & $\mathrm{M}$ \\
\hline $\mathrm{N}$ & 103 & 99 \\
Media & 30,91 & 33,56 \\
Nivel & Promedio & Promedio \\
Mediana & 31,00 & 34,00 \\
Desv. Típ. & 5,139 & 5,081 \\
Varianza & 26,414 & 25,821 \\
\hline
\end{tabular}

$\mathrm{H}=$ Hombre, $\mathrm{M}=$ Mujer

En la Tabla 7, se observa que tanto varones como mujeres obtienen el nivel Nivel Promedio. 
Tabla 8. Resultados en factores personales de resiliencia según grado de instrucción

\begin{tabular}{|c|c|c|c|c|c|c|c|c|c|c|}
\hline & & utoestim & Emp & tía & utonomía & Humor & Creat & vidad & & \\
\hline & 5to & 6to & 5to & 6to & 5 to & 6to & 5to & 6to & 5to & 6to \\
\hline $\mathrm{N}$ & 72 & 130 & 72 & 130 & 72 & 130 & 72 & 130 & 72 & 130 \\
\hline Media & 8,19 & 7,62 & 7,31 & 7,08 & 7,28 & 6,59 & 5,83 & 5,78 & 4,89 & 4,49 \\
\hline Nivel & $\begin{array}{l}\text { Me- } \\
\text { dio }\end{array}$ & $\begin{array}{c}\text { Me- } \\
\text { dio }\end{array}$ & $\begin{array}{l}\text { Me- } \\
\text { dio }\end{array}$ & $\begin{array}{l}\text { Me- } \\
\text { dio }\end{array}$ & $\begin{array}{l}\text { Me- } \\
\text { dio }\end{array}$ & $\begin{array}{c}\text { Me- } \\
\text { dio }\end{array}$ & $\begin{array}{l}\text { Me- } \\
\text { dio }\end{array}$ & $\begin{array}{c}\text { Me- } \\
\text { dio }\end{array}$ & $\begin{array}{l}\mathrm{Me}- \\
\text { dio }\end{array}$ & $\begin{array}{c}\mathrm{Me}- \\
\text { dio }\end{array}$ \\
\hline Mediana & 9,00 & 8,00 & 8,00 & 7,00 & 7,00 & 7,00 & 6,00 & 6,00 & 5,00 & 4,00 \\
\hline Desv. Típ & 1,553 & 1,869 & 1,851 & 1,826 & 1,672 & 1,799 & 1,434 & 1,614 & 1,525 & 1,423 \\
\hline Varianza & 2,412 & 3,492 & 3,426 & 3,334 & 2,795 & 3,236 & 2,056 & 2,604 & 2,326 & 2,026 \\
\hline
\end{tabular}

La Tabla 8 indica que el promedio de alumnos de $5 .{ }^{\text {to }}$ grado es mayor que los alumnos de $6 .{ }^{\text {to }}$ grado, en todos los factores personales de resiliencia (Autoestima, Empatía, Autonomía, Humor y Creatividad). Al comparar los niveles asociados a las medias obtenidas en todos los casos corresponden al nivel medio.

\section{Resultados Inferenciales}

Tabla 9. Diferencia de medias en resiliencia, según sexo

\begin{tabular}{|c|c|c|}
\hline & \multicolumn{2}{|c|}{ RESILIENCIA } \\
\hline & $\mathrm{H}$ & M \\
\hline $\mathrm{N}$ & 103 & 99 \\
\hline Media & 30,91 & 33,56 \\
\hline U de Mann-Whitney & & \\
\hline $\mathrm{Z}$ & & \\
\hline Sig. asintót. (bilateral) & & \\
\hline
\end{tabular}

La Tabla 9 presenta el estadístico U de Mann-Whitney, además la tipificación (Z) de la Resiliencia, y el nivel crítico bilateral (Sig.) el cual es menor a 0,05 . Por tanto, podemos rechazar la hipótesis de igualdad de promedios y concluir que los grupos definidos por la variable sexo proceden de poblaciones con distinto promedio. Esto indica que las mujeres obtienen mayor promedio en Resiliencia, de modo significativo, en relación a los hombres.

Tabla 10. Diferencia de medias en factores personales de resiliencia, según sexo

\begin{tabular}{lcccccccccc}
\hline & \multicolumn{2}{c}{ Autoestima } & \multicolumn{2}{c}{ Empatía } & \multicolumn{2}{c}{ Autonomía } & \multicolumn{2}{c}{ Humor } & \multicolumn{2}{c}{ Creatividad } \\
\hline & $\mathrm{H}$ & $\mathrm{M}$ & $\mathrm{H}$ & $\mathrm{M}$ & $\mathrm{H}$ & $\mathrm{M}$ & $\mathrm{H}$ & $\mathrm{M}$ & $\mathrm{H}$ & $\mathrm{M}$ \\
\hline $\mathrm{N}$ & 103 & 99 & 103 & 99 & 103 & 99 & 103 & 99 & 103 & 99 \\
Media & 7,62 & 8,04 & 6,62 & 7,73 & 6,71 & 6,97 & 5,66 & 5,95 & 4,30 & 4,87 \\
U de Mann-Whitney & 4317,500 & 3344,000 & 4616,500 & 4613,500 & 3977,000 \\
Z & $-1,914$ & $-4,288$ & $-1,177$ & $-1,197$ & $-2,755$ \\
Sig.asintót(bilateral) &, 056 & $\mathbf{, 0 0 0}$ &, 239 & \multirow{2}{*}{, 231} & $\mathbf{0 0 6}$ \\
\hline
\end{tabular}


La tabla 10 presenta el estadístico U de Mann-Whitney, además la tipificación (Z) de los factores personales de resiliencia, y el nivel crítico bilateral (Sig.) el cual es menor a 0,05 en el caso de Empatía y Creatividad. Por tanto, podemos rechazar la hipótesis de igualdad de promedios y concluir que los grupos definidos por la variable sexo proceden de poblaciones con distinto promedio. Esto indica que las mujeres obtienen mayores promedios en Empatía y Creatividad, de modo significativo, en relación a los hombres.

Tabla 11. Diferencia de medias en resiliencia, según grado de estudio

\begin{tabular}{ccc}
\hline & \multicolumn{3}{c}{ RESILIENCIA } \\
\hline $\mathrm{N}$ & 5 to. & 6 to. \\
Media & 72 & 130 \\
$\mathrm{U}$ de Mann-Whitney & 33,50 & 31,49 \\
$\mathrm{Z}$ & & 3703,500 \\
Sig. asintót. (bilateral) & $-2,458$ & \\
\hline
\end{tabular}

La Tabla 11, presenta el estadístico U de Mann-Whitney, además la tipificación (Z) de la Resiliencia, y el nivel crítico bilateral (Sig.) el cual es menor a 0,05. Por tanto, podemos rechazar la hipótesis de igualdad de promedios y concluir que los grupos definidos por la variable grado de estudio proceden de poblaciones con distinto promedio. Esto indica que los alumnos de $5 .{ }^{\text {to }}$ grado obtienen mayor promedio en Resiliencia, de modo significativo, en relación a los alumnos de $6 .{ }^{\text {to }}$ grado.

\begin{tabular}{lcccccccccc}
\hline \multicolumn{10}{c}{ Tabla 12. Diferencia de medias en factores personales de resiliencia, según grado de estudio } \\
\hline \multicolumn{7}{c}{ Autoestima } & Empatía & Autonomía & Humor & Creatividad \\
\hline & 5 to. & 6 to. & 5 to. & 6 to & 5 to. & 6 to & 5 to. & 6 to & 5 to. & 6 to \\
N & 72 & 130 & 72 & 130 & 72 & 130 & 72 & 130 & 72 & 130 \\
Media & 8,19 & 7,62 & 7,31 & 7,08 & 7,28 & 6,59 & 5,83 & 5,78 & 4,89 & 4,41 \\
U de Mann-Whitney & 3888,500 & 4305,500 & 3661,000 & 4561,500 & 3785,500 \\
Z & $-2,025$ &,- 955 & $-2,598$ &,- 305 & $-2,293$ \\
Sig. asintót. (bilateral) &, 043 &, 339 &, 009 &, 760 &, 022 \\
\hline
\end{tabular}

La Tabla 12, presenta el estadístico U de Mann-Whitney, además la tipificación (Z) de los factores personales de resiliencia, y el nivel crítico bilateral (Sig.) el cual es menor a 0,05 en el caso de Autoestima, Autonomía y Creatividad. Por tanto, en estos casos, podemos rechazar la hipótesis de igualdad de promedios y concluir que los grupos definidos por la variable grado de estudios proceden de poblaciones con distinto promedio. Esto indica que los alumnos de 5 ${ }^{\text {to }}$ grado obtienen mayores promedios en Autoestima, Autonomía y Creatividad, de modo significativo, en relación a los alumnos de $6 .{ }^{\text {to }}$ grado.

La Tabla 13, presenta el estadístico U de Mann-Whitney, además la tipificación 
Tabla 13. Diferencia de medias en rendimiento académico, según sexo

\begin{tabular}{lcccccccc}
\hline & \multicolumn{2}{c}{ Comunicación } & \multicolumn{2}{c}{ Matemática } & \multicolumn{2}{c}{ Ciencia y A. } & \multicolumn{2}{c}{ Personal S } \\
& H & M & H & M & H & M & H & M \\
\hline N & 103 & 99 & 103 & 99 & 103 & 99 & 103 & 99 \\
Media & 2,28 & 2,58 & 2,37 & 2,48 & 2,29 & 2,49 & 2,42 & 2,70 \\
U de Mann-Whitney & 3822,500 & 4704,000 & 4278,000 & 3922,500 \\
Z & $-3,419$ & $-1,046$ & $-2,225$ & & $-3,213$ \\
Sig. asint (bilateral) &, 001 & &, 295 & &, 026 & &, 001 \\
\hline
\end{tabular}

(Z) de las áreas de rendimiento académico, y el nivel crítico bilateral (Sig.) es menor a 0,05 en el caso de Comunicación Integral, Ciencia y Ambiente y Personal Social. Por tanto, podemos rechazar la hipótesis de igualdad de promedios y concluir que los grupos definidos por la variable sexo proceden de poblaciones con distinto promedio. Esto indica que las mujeres obtienen mayores promedios en Comunicación Integral, Ciencia y Ambiente y Personal Social, de modo significativo, en relación a los hombres.

Tabla 14. Diferencia de medias en rendimiento académico, según grado de instrucción

\begin{tabular}{|c|c|c|c|c|c|c|c|c|}
\hline & \multicolumn{2}{|c|}{ Comunic. } & \multicolumn{2}{|c|}{ Matem. } & \multicolumn{2}{|c|}{ Ciencia } & \multicolumn{2}{|c|}{ Person. } \\
\hline & 5to. & 6to & 5to. & 6to. & 5to. & 6to & 5to. & 6to \\
\hline $\mathrm{N}$ & 72 & 130 & 72 & 130 & 72 & 130 & 72 & 130 \\
\hline Media & 2,43 & 2,42 & 2,50 & 2,38 & 2,33 & 2,42 & 2,65 & 2,50 \\
\hline U de Mann-Whitney & \multicolumn{2}{|c|}{4591,500} & \multicolumn{2}{|c|}{4382,000} & \multicolumn{2}{|c|}{4267,500} & \multicolumn{2}{|c|}{4232,500} \\
\hline Z & \multicolumn{2}{|c|}{,- 248} & \multicolumn{2}{|c|}{,- 825} & \multicolumn{2}{|c|}{$-1,167$} & \multicolumn{2}{|c|}{$-1,276$} \\
\hline Sig. asintót. (bilateral & \multicolumn{2}{|c|}{805} & \multicolumn{2}{|c|}{,409 } & \multicolumn{2}{|c|}{243} & \multicolumn{2}{|c|}{,202 } \\
\hline
\end{tabular}

La Tabla 14, presenta el estadístico U de Mann-Whitney, además la tipificación (Z) de las áreas de rendimiento académico, y el nivel crítico bilateral (Sig.) es mayor a 0,05 en todos los casos. Por tanto, no podemos rechazar la hipótesis de igualdad de promedios y concluir que los grupos definidos por la variable grado de estudio proceden de poblaciones con similar promedio. Esto indica que no existen diferencias significativas en las áreas del rendimiento académico según el grado de estudio. 
Tabla 15. Resultados en coeficiente de correlación de Spearman entre factores personales de resiliencia y áreas de rendimiento académico

\begin{tabular}{llcccc}
\hline & & Comunicación & Matemática & Ciencia y A. & Personal S \\
\hline Autoestima & Rho de Spearman &, $188^{* *}$ &, $193^{* *}$ &, $166^{*}$ &, $207^{* *}$ \\
\hline & Sig. (bilateral) &, 008 &, 006 &, 019 &, 003 \\
& $\mathrm{~N}$ & 202 & 202 & 202 & 202 \\
\hline Empatía & Rho de Spearman &, $200^{* *}$ &, $152^{*}$ &, $244^{* *}$ &, $195^{* *}$ \\
\hline Autonomía & Sig. (bilateral) &, 004 &, 031 &, 000 &, 006 \\
& $\mathrm{~N}$ & 202 & 202 & 202 & 202 \\
\hline \multirow{5}{*}{ Humor } & Rho de Spearman &, $216^{* *}$ &, $174 *$ &, 133 &, $221^{* *}$ \\
\hline & Sig. (bilateral) &, 002 &, 013 &, 059 &, 002 \\
& $\mathrm{~N}$ & 202 & 202 & 202 & 202 \\
\hline Creatividad & Rho de Spearman &, $185^{* *}$ &, 083 &, $162 *$ &, $139 *$ \\
\hline & Sig. (bilateral) &, 008 &, 242 &, 021 &, 048 \\
& $\mathrm{~N}$ & 202 & 202 & 202 & 202 \\
\hline Rho de Spearman &, 060 &, 086 &, 037 &, $189 * *$ \\
\hline & Sig. (bilateral) &, 393 &, 226 &, 597 &, 007 \\
& $\mathrm{~N}$ & 202 & 202 & 202 & 202 \\
\hline & Rho de Spearman &, $277^{* *}$ &, $235^{* *}$ &, $253^{* *}$ &, $326^{* *}$ \\
\hline & Sig. (bilateral) &, 000 &, 001 &, 000 &, 000 \\
& $\mathrm{~N}$ & 202 & 202 & 202 & 202 \\
\hline
\end{tabular}

**. La correlación es significativa al nivel 0,01 (bilateral).

*. La correlación es significante al nivel 0,05 (bilateral).

En la Tabla 15, se observan correlaciones significativas entre los factores Autoestima, Empatía, con las áreas de rendimiento académico, en el caso de Resiliencia también presenta una correlación con el rendimiento académico presentando una correlación más alta con el área personal social. El factor personal Autonomía presenta correlación significativa con Comunicación Integral, Matemática y Personal Social; el factor personal Humor presenta correlación con Comunicación Integral, Ciencia-Ambiente y Personal Social; en el caso de Creatividad obtiene correlación con el área Personal Social.

\section{DISCUSIÓN}

Los estudiantes de la muestra obtienen un nivel de resiliencia promedio con lo cual se comprueba la primera hipótesis específica de la investigación, ya que el mayor porcentaje de los alumnos se ubica entre el nivel promedio y el nivel alto de resiliencia (Véase Tabla 3).

Así mismo, obtienen un nivel promedio en los factores personales de resiliencia con lo cual se comprueba la segunda hipótesis específica de la investigación, ya que la mayoría se ubicaba en el nivel medio en los factores de resiliencia y en el nivel alto en empatía (Véase Tabla 4) 
Al realizar un análisis del rendimiento académico de los alumnos de 5to. y 6to. grado de primaria se halló que la mayoría de los alumnos se encuentran en el nivel B lo que significa que los alumnos se encuentran en proceso (Véase Tabla 2). Este hallazgo demuestra que los alumnos de la muestra no han obtenido los logros previstos en las áreas del rendimiento académico esperado para su grado de estudios. Esto permitió rechazar la hipótesis específica $\mathrm{N}{ }^{\circ} 3$.

Los niveles de resiliencia resultaron significativamente diferentes según la variable sexo, comprobando así la cuarta hipótesis específica de este estudio, ya que existen diferencias significativas en los niveles de resiliencia de varones y mujeres a favor de las mujeres. (Véase Tabla 9).

Estos resultados coinciden con los de Álvarez y Cáceres (2010), quienes en un estudio sobre resiliencia realizado en Colombia encontraron también diferencias significativas en favor de las mujeres en la capacidad de resiliencia. Por otra parte, Álvarez y Cáceres (2010) en su estudio "Resiliencia, rendimiento académico y variables sociodemográficas en estudiantes universitarios de Bucaramanga (Colombia)"; también encuentran que las mujeres resultaron ser más resilientes que los hombres. Así Aguirre (2004) en el estudio "Capacidad y factores asociados a la resiliencia en adolescentes del C.E. Mariscal Andrés Avelino Cáceres del sector IV de Pamplona Alta San Juan de Miraflores 2002", en adolescentes de 12 a 16 años, hombres y mujeres; encuentra también que las mujeres tienen mayor capacidad de resiliencia. Estos resultados también coinciden con lo de Matalinares et al. (2011), quienes también encontraron diferencias significativas en el factor de resiliencia empatía a favor de las mujeres.

Una explicación a tal situación hace suponer que los recursos de adaptación a situaciones estresantes en las mujeres son más efectivos en cuanto ellas se interesan menos por competir y lograr; al parecer, el pertenecer al género femenino es considerado como una variable protectora, según lo indican estudios que han observado una mayor vulnerabilidad al riesgo en los hombres. Rutter (1990) alude al género masculino como una variable que genera una mayor vulnerabilidad al riesgo, y da cuenta de los mecanismos que subyacen a esta característica: así explica que los varones estarían más expuestos que las mujeres a experimentar situaciones de riesgo en forma directa; en situaciones de quiebre familiar, los niños tienen más probabilidad que las niñas de ser reubicados en algunas institución; los niños tienden a reaccionar a través de conductas oposicionistas con mayor frecuencia que las niñas, lo cual a su vez genera respuestas negativas de parte de los padres; por último, afirma el citado autor que, en general, las personas tienden a interpretar de modo distinto las conductas agresivas de los niños que las de las niñas y a su vez, a castigar más severamente estos comportamientos en los varones. La acción de las hormonas sexuales conforma redes neurales y procesos bioquímicos diferentes en los cerebros de hombres y mujeres, ya desde los primeros meses de vida intrauterina. Pero también las experiencias y aprendizajes en los contextos socioculturales conforman y organizan el cerebro de cada persona originando capacidades y comportamientos propios y diferenciales (García, 2003). 
$\mathrm{Al}$ analizar los resultados comparativos en los factores personales de resiliencia según la variable sexo, se encuentran diferencias significativas, ya que los resultados muestran que las mujeres obtienen mayores promedios en empatía y creatividad en relación a los hombres de modo significativo, comprobándose así la quinta hipótesis específica de la investigación (Véase Tabla 10).

Existen diferencias significativas en resiliencia según el grado de estudio a favor de los alumnos de 5to. grado comprobándose la hipótesis número seis de esta investigación, pues los alumnos de $5^{\circ}$ grado obtienen mayor promedio en resiliencia, de modo significativo, que los de $6^{\circ}$ grado, no encontrándose antecedentes semejantes en la literatura (Véase tabla 11).

Estos hallazgos discrepan con los de Velásquez y Montgomery (2009), quienes en el estudio "Resiliencia y depresión en estudiantes de secundaria de Lima Metropolitana con y sin participación en actos violentos" encuentran que el grado de instrucción no influye en la resiliencia. De igual forma, con los de Bulnes et. al, (2008), en el estudio "Resiliencia y estilos de socialización parental en escolares de 4to y 5to año de secundaria de Lima Metropolitana" se realizan análisis comparativos de la variable resiliencia entre escolares según grado de estudio, para el cuarto año de secundaria y quinto año de secundaria, no encontrando diferencias significativas entre ambos grupos. Los resultados difieren también con el estudio de Saavedra y Villalta (2008), en el estudio "Medición de las características resilientes, un estudio comparativo en personas entre 15 y 65 años" encuentran que los niveles de resiliencia no están asociados directamente a los tramos de edad, ya que se obtienen resultados similares en las diferentes etapas de vida.

Los interesantes resultados de la investigación, en torno a las diferencias significativas en resiliencia en función al grado, se explican en las diferencias en las etapas cronológicas y psicológicas por las que atraviesan los alumnos de quinto y sexto grado respectivamente; es decir, los estudiantes de 5to. Grado son diferentes a los de 6to. en cuanto los primeros se encuentran más cerca a una etapa de vida de en la que el equilibrio emocional y las exigencias académicas favorecen respuestas académicas y emocionales más óptimas que los estudiantes de 6to. Grado, los que en su gran mayoría se encuentran en una etapa biológica de recambios hormonales, que innegablemente repercuten en el sistema nervioso, controlador de los estados emocionales y las respuestas afectivas aunadas a las presiones y expectativas de pasar a un nivel de instrucción superior, con las consiguientes presiones académicas y familiares que conlleva esta etapa transitoria, todo lo cual conlleva a repercusiones emocionales, comportamentales y de rendimiento académico posiblemente a desfavor.

Se encuentra que los alumnos de 5to. grado obtienen mayores promedios en Autoestima, Autonomía y Creatividad que los de 6to. grado en modo significativo, comprobándose la hipótesis número siete de esta investigación, ya que existen diferencias significativas en los factores personales de resiliencia de los estudiantes según variable grado de instrucción (Véase Tabla 12). 
En lo que respecta al rendimiento académico según la variable sexo, las mujeres obtienen mayores promedios en Comunicación Integral, Ciencia y Ambiente y Personal Social, de modo significativo, en relación a los hombres. Estos resultados concuerdan con los de Alcaide (2009), quien en el estudio "Autoconcepto y rendimiento académico en alumnos de 1ro. de bachillerato según el género", también encuentra que las mujeres obtienen mejores resultados académicos que los hombres. Así Di Gresia, Porto y Ripani (2002) en el estudio "Rendimiento de los Estudiantes de las Universidades Públicas Argentinas” encuentran en 29 universidades que los varones tienen menor rendimiento en los estudios que las mujeres. Los resultados hallado concuerdan también con los de Peralta, Ramírez y Castaño (2006), quienes en el estudio "Factores resilientes asociados al rendimiento académico en estudiantes pertenecientes a la Universidad de Sucre (Colombia)" encontraron que los hombres presentaban mayores problemas académicos que las mujeres y que las mujeres presentaban rendimientos académicos más altos que los hombres, pero que estas evidencias no alcanzaron a ser diferencias estadísticamente significativas .

Ahora bien, una posible explicación a estas diferencias en el rendimiento académico según sexo a favor de las mujeres, se podría deber a que probablemente, las mujeres estén más motivadas para el trabajo académico y/o para desarrollar habilidades académicas. Esto lleva a comprobar nuestra octava hipótesis específica de la investigación (Véase tabla 13).

En la presente investigación los alumnos de 5to grado obtienen mejores promedios que los alumnos de 6to., en las áreas de rendimiento académico, a excepción de Ciencia y Ambiente, éstas diferencias no eran estadísticamente significativas en las áreas del rendimiento académico según el grado de estudio. (Véase tabla 14). Por ello se rechaza la novena hipótesis específica, ya que los resultados mostraron que no existen diferencias significativas en las áreas de rendimiento académico según el grado de estudios.

Resultados que discrepan con lo hallado por Peralta, Ramírez y Castaño (2006) quienes al investigar los "Factores resilientes asociados al rendimiento académico en estudiantes pertenecientes a la Universidad de Sucre (Colombia)", en la variable de semestres en curso, por ejemplo, se encontraron que gran parte de los que cursan tercer, cuarto y quinto semestre poseen dificultades, debido a que existe mayor número de estudiantes con bajo rendimiento académico; después, en los semestres superiores, el rendimiento tiende a mejorar. Esto puede deberse a que existe mayor exigencia en los primeros semestres, porque hay más contenidos teóricos que prácticos, que apuntan al desarrollo disciplinar de la carrera; en cambio, los últimos semestres se caracterizan por ser más prácticos, debido a que los contenidos son en la mayoría de aplicación.

Los resultados reconfirman que el aprendizaje de contenidos escolares es un proceso sumamente complejo, que depende de innumerables factores que coovarían entre sí; de igual modo los mismos, deberían alertar a los profesionales de la educación 
sobre los riesgos de realizar generalizaciones amplias que llevan a suponer que la resiliencia se encuentra asociada al desempeño académico en forma generalizada. Las evidencias sugieren que la resiliencia es más elevada en los alumnos de 5to. Grado que en los de 6to. Y que encontraremos alumnos de 6to. Grado que sin embargo presenten un rendimiento académico igual o mayor a los de 5to.

Los análisis estadísticos permitieron establecer que la resiliencia presenta una correlación significativa con todas las áreas del rendimiento académico: Comunicación Integral, Lógico Matemático, Ciencia y Ambiente y Personal Social; dándose en ésta última área la correlación más alta. (Véase tabla 15). Los niveles de correlación, son estadísticamente significativos y por ello podemos hablar de la existencia de una tendencia general que indicaría que a medida que aumentan los niveles de resiliencia total de un sujeto, aumenta el rendimiento académico en Matemáticas, Comunicación Integral, Ciencia y Ambiente y Personal Social.

Los resultados de la presente investigación coinciden con los de Peralta, Ramírez y Castaño (2006) quienes en su estudio "Factores resilientes asociados al rendimiento académico en estudiantes pertenecientes a la universidad de Sucre (Colombia)", hallaron que existen diferencias estadísticamente significativas de los factores resilientes entre los estudiantes con alto y bajo rendimiento académico, y este último grupo es el que se encuentra en mayor riesgo debido a que existen menos factores que se asocian a él. Encontraron además que la iniciativa, entre todos los demás factores resilientes, es determinante en el rendimiento académico alto o bajo de los estudiantes en esta universidad pública, mientras que los otros factores no presentaron significancia estadística. Por último, encontraron que existen, diferencias estadísticamente significativas entre los estudiantes de bajo y alto rendimiento que se encuentran en las variables de introspección, interacción, iniciativa, independencia, moralidad y pensamiento crítico. Son los estudiantes con buen rendimiento académico los que tienden a tener puntajes más altos en el cuestionario de resiliencia, lo que implica mayor presencia de estos factores en este grupo.

Así mismo, los resultados de la presente investigación también coinciden con los de Villalta (2010), quien en su estudio "Factores de resiliencia asociados al rendimiento académico en estudiantes de contextos de alta vulnerabilidad social" en alumnos de educación media de la región metropolitana de Chile, encuentra que la relación entre resiliencia y logros de aprendizaje se fortalece en dos situaciones de adversidad que reportan los adolescentes: divorcio o separación de sus padres, y embarazo propio o de la pareja. Es decir, conforme aumenta la percepción negativa de la calidad de vida, baja la puntuación de resiliencia. Adicionalmente, se encuentra correlación positiva moderada y significativa entre el promedio de notas y la puntuación general de resiliencia.

Por lo tanto, se acepta la hipótesis general de esta investigación porque se halló que existe relación entre los factores personales de resiliencia y el rendimiento académico en los alumnos del quinto y sexto grado de primaria en la muestra. 
Ahora bien, al analizar cada uno de los factores personales de resiliencia, se encontró que el factor autoestima, entendida como la valoración que el niño tiene sobre sí mismo a partir de las ideas y sentimientos que se derivan de su propio autoconocimiento, así como de las influencias y mensajes que recibe de otras personas y también de su medio sociocultural Regional Training (1999) citado por Salgado (2005) correlaciona significativamente con todas las áreas del rendimiento académico: Comunicación Integral. Lógico Matemático, Personal Social y Ciencia y Ambiente. (Véase Tabla 15).

En el caso del factor empatía, conceptualizada por Hoffman (1983) citado por Salgado (2005) como la experiencia afectiva vicaria de los sentimientos de otra persona; también definida como la habilidad que posee un individuo de inferir los pensamientos y sentimientos de otros, lo que genera sentimientos de simpatía, comprensión y ternura, correlaciona significativamente con Comunicación Integral, Lógico Matemático, Personal Social y Ciencia y Ambiente. (Véase Tabla 15).

De otro lado el factor autonomía, entendida como la capacidad del niño de decidir y realizar independientemente acciones que guarden consonancia con sus intereses y posibilidades de acuerdo a su momento de desarrollo. correlaciona significativamente con Comunicación Integral, Matemática y Personal Social en Panez (2002) citado por Salgado (2005) (Véase Tabla 15).

El factor humor, referido a la disposición del espíritu a la alegría, permite alejar del foco de tensión, relativizar, positivizar, elaborar de un modo lúdico, encontrar lo cómico entre la tragedia. correlaciona significativamente con Comunicación Integral, Ciencia-Ambiente y Personal Social (Wolin \& Wolin, 1993 citado por Salgado, 2005 (Véase tabla 15).

Por último, el factor creatividad, que es la capacidad del niño para transformar o construir palabras, objetos, acciones en algo innovador o de manera innovadora en relación a los patrones de referencia de su grupo; esta definición enfatiza la apreciación y solución innovadora y eficaz de las situaciones, esto es, acercarse y apreciar los problemas desde una perspectiva y dar soluciones que tengan un carácter novedoso y eficaz (Regional Training, 1999 citado por Salgado, 2005) correlaciona significativamente con personal social. (Véase tabla 15).

Finalmente, debería tenerse mucho cuidado al suponer que un mejoramiento en la resiliencia podría beneficiar los aprendizajes sin considerar el sexo, curso y grado de estudio en los estudiantes al momento de elaborar programas psicológicos en pro de una mejora de la calidad educativa.

\section{CONCLUSIONES}

1. Los factores personales Autoestima y Empatía presentan correlación significativa con todas las áreas del rendimiento académico: Comunicación Integral, Lógico Matemático, Personal Social y Ciencia y Ambiente. Resiliencia presenta correlación significativa con todas las áreas de rendimiento académico: 
Comunicación Integral, Lógico Matemático, Personal Social y Ciencia y Ambiente. El factor personal Autonomía presenta correlación significativa con Comunicación Integral, Lógico Matemático y Personal Social. El factor personal Humor presenta correlación significativa con Comunicación Integral, Ciencia-Ambiente y Personal Social. En el caso de Creatividad obtiene correlación significativa con Personal Social.

2. En Resiliencia el mayor porcentaje de estudiantes obtiene nivel promedio.

3. En los factores personales de resiliencia los estudiantes obtienen con más frecuencia y porcentaje el nivel Medio, a excepción del factor Empatía donde en mayor porcentaje se encuentran en Nivel Alto.

4. En Comunicación Integral, Matemática y Personal Social, los estudiantes obtienen Nivel B (En Proceso). En Ciencia y Ambiente el nivel A (Logro previsto) presenta mayor porcentaje.

5. En Resiliencia, existen diferencias significativas según la variable sexo a favor de las mujeres.

6. En los Factores Personales de Resiliencia, según la variable sexo, existen diferencias significativas en Empatía y Creatividad a favor de las mujeres.

7. En Resiliencia según la variable grado de instrucción, existen diferencias significativas a favor de los alumnos de 5to. grado.

8. En los Factores Personales de Resiliencia, según la variable grado de instrucción, existen diferencias significativas en Autoestima, Autonomía y Creatividad, a favor de los alumnos de 5to. grado.

9. El rendimiento académico en Comunicación Integral, Ciencia y Ambiente y Personal Social es significativamente más alto en las mujeres que en los hombres.

10. En rendimiento académico no se encontraron diferencias significativas, según la variable grado de instrucción.

\section{REFERENCIAS BIBLIOGRÁFICAS}

Adell, M. (2002).Estrategias para mejorar el rendimiento académico de los adolescentes. Madrid: Ediciones Pirámide.

Adell, M. A. (2006). Estrategias para mejorar el rendimiento académico de los adolescentes. Madrid: Pirámide.

Aguirre (2004). Capacidad y factores asociados a la resiliencia, en adolescentes del C.E. Mariscal Andrés Avelino Cáceres del Sector IV de Pamplona Alta San Juan de Miraflores 2002. Tesis para optar el Título de Licenciado en Enfermería UNMSM. Obtenida el 21 de febrero del 2011 de: http://sisbib.unmsm.edu.pe/bibvirtualdata/ tesis/salud/aguirre_ga/T_completo.PDF 
Astete, A.; Benavides, G. y Palacios, E. (2009). Resiliencia en niños de 8 a 12 años provenientes de familias disfuncionales. (Tesis de Licenciatura no publicada). Lima: Universidad Peruana Cayetano Heredia.

Aponte, D. (2009). Relación entre estilos de crianza parental y resiliencia adolescente en la conducta violenta. Tesis para optar el grado académico de Psicólogo UNMSM.

Bulnes,M., Ponce,C., Huerta,R., Álvarez,C., Santibáñez,W., Atalaya,M., Aliaga,J., Morocho,J. (2008); Resiliencia y estilos de socialización parental. Revista de investigación en Psicología, Vol.11, No .2,2008, Págs. 67-91. Obtenida el 10 de febrero del 2011, en sisbib, de: http://sisbib.unmsm.edu.pe/BVRevistas/ Investigacion_Psicologia/v11_n2/pdf/a06v11n2.pdf

Di Gresia, L., Porto A., Ripani L (2002). Rendimiento de los estudiantes de las universidades públicas argentinas. Universidad Nacional de la Plata. Facultad de Ciencias Económicas. Obtenida el 12 de julio del 2011 de: http://www.depeco.econo. unlp.edu.ar/doctrab/doc45.pdf

Escudero, J. (2007). Relación entre resiliencia y sentido de vida (religiosidad intrínseca y objetivos vitales) en adolescentes de 4to. y 5to. de secundaria. Tesis para optar el grado académico de Psicólogo, UNMSM.

Flores, M. (2008). Resiliencia y Proyecto de vida en estudiantes del 3er. Año de secundaria de la UGEL 03. Tesis para grado académico de magíster UNMSM.

García, E. (2003). Neuropsicología y género. Revista de la Asociación Española de Neuropsiquiatría n.86 Madrid abr.-jun. 2003. Obtenida el 25 de setiembre del 2011 de: http://scielo.isciii.es/scielo.php?script $=$ sci_arttext\&pid $=$ S0211-57352003000200002

Ibañez, B. (1995). Manual para la elaboración de tesis. (2da. edición). México: Trillas

Kalawski, J. y Haz, A. (2003) Y... ¿dónde está la Resiliencia? Una reflexión conceptual. Revista Interamericana de Psicologia Vol. 37, Num. 2 pp. 365-372 http://www. paho.org/Spanish/HPP/HPF/ADOL/Resil6x9.pdf

Matalinares, M. Arenas, C. Yaringaño, J. Sotelo, L. Sotelo, N. Díaz, G. Dioses, A. Ramos, R. Mendoza, P. Medina, Y. Pezua, M. Muratta, R. Pareja, C. y Tipacti, R. (2011) Factores personales de resiliencia y autoconcepto en estudiantes de primaria de Lima Metropolitana. Revista de Investigaciones Psicológicas. Vol. 14 - N. ${ }^{\circ} 1$ pp. 187-207.

Melillo, A. Suárez, E. (2001). Resiliencia. Descubriendo las propias fortalezas. Buenos Aires: Paidós. Págs. 19, 20, 21, 22, 30, 32, 33, 34, 35, 88

Quintana, Montgomery y Malaver (2009) Modos de Afrontamiento y Conducta Resiliente en adolescentes espectadores de violencia entre pares. Revista de Investigación en Psicología Vol. 12, $\mathrm{N}^{0}$ 1, 2009. Obtenida el 10 de febrero del 2011, en sisbib de: http://sisbib.unmsm.edu.pe/BVRevistas/Investigacion_Psicologia/ v12_n1/pdf/ a11v12n1.pdf

Rutter, M. (1999). Resilience concepts and findings: implications for family therapy.

Journal of Family Therapy, 21, pp. 119-144.Saavedra y Villalta (2008). Medición de las características resilientes un estudio comparativo en personas entre 15 y 65 años. 
Liberabit: Lima (Perú) 14: 31-40, 2008. ISSN: 1729 - 4827. Obtenida el 20 de marzo del 2011 de: http://www.scielo.org.pe/pdf/liber/v14n14/a05v14n14.pdf

Salgado, A. (2005). Métodos e instrumentos para medir la resiliencia una alternativa peruana. Liberabit. Revista de Psicología. Universidad de San Martín de Porres. Obtenida el 15 de mayo del 2011 de: http://redalyc.uaemex.mx/pdf/686/68601106.pdf

Salgado, A. (2011). Felicidad, resiliencia y optimismo en estudiantes de colegios nacionales de la ciudad de Lima. Liberabit, Vol. 15, N. ${ }^{\circ} 2$, 2009. 133-141. Obtenida el 15 de febrero del 2011, en dialnet de : http://dialnet.unirioja.es/servlet/ articulo? codigo $=3124724$

Velásquez y Montgomery (2009). Resiliencia y depresión en estudiantes de secundaria de Lima Metropolitana con y sin participación en actos violentos. Revista de Investigación en Psicología, Vol.12, $\mathrm{N}^{\mathrm{o}}$ 2, 2009, Págs. 75-78 Obtenida el 15 de febrero del 2011, en dialnet, de: http://dialnet.unrioja.es/servlet/articulo?codigo $=3268371 \& i n f o=r e$ sumen\&modo $=$ popup

Villalta, M. (2010) Relación entre factores de resiliencia y rendimiento académico en alumnos adolescentes de establecimientos educativos ubicados en contextos de alta vulnerabilidad social. Obtenida el 2 de abril del 2011, en: http://www.scielo.org. ve/pdf/p/v31n88/art07.pdf 
